\title{
NUMERICAL SOLUTION OF LINEAR-QUADRATIC OPTIMAL CONTROL PROBLEMS FOR SWITCHING SYSTEMS
}

\author{
SHAHLAR MEHERREM, DENIZ H. GUCOGLU, AND SAMIR GULIYEV
}

Received 10 July, 2017

\begin{abstract}
In this paper we discuss the approach for optimal switching control problem with unknown switching points. The case with unknown switching point is more general and generalizes the results existing in the literature. By using suitable transformation, the main problem is reduced into a problem with known interval and further the unknown boundary of the integral in the minimization functional is reduced to the known one. This fact is illustrated by an example. The reduced problem is solved numerically by using the Gradient Projection Method Algorithm.
\end{abstract}

2010 Mathematics Subject Classification: 49K15; 49M30; 93C05

Keywords: optimal control, switching system, numerical solution, finite approximation

\section{INTRODUCTION}

There are many articles dedicated to the Linear-Quadratic Optimal Control Problems (LQOCPs) for switching systems. These problems are particular kind of hybrid systems. Examples of switching systems can be found in the area of engineering, chemical processes, automotive systems and military services. The published results in the literature can be mainly classified into two categories; one is theoretical $[3,4,6,7,9,10,16-19,22]$ and practical $[2,8,11,13-15,20,21]$. The very earliest result which is proved a maximum principe for hybrid system for autonomous switching system is in [20]. More theoretical results of the maximum principle are obtained by Picolli in [18] and Sussman in [19] which are correspondingly is called hybrid maximum principle and maximum principle for the hybrid system in the case of the minimization functional is non-smooth. In [6, 22] switching systems are investigated by using dynamical programming approach to derive Hamilton-Jacobi-Belmann equations. But there are some practical results for the switching optimal control problem which has significant applications to real-world problems. In [5] conceptual algorithms were given for general hybrid optimal control problems. In [13], for a class of discrete-time hybrid system an algorithm is given by using constrained differential programming approach by author. An application to power train control can be found in [1]. Some heuristically oriented methods have been reported in [12], which used algorithms pruning the search trees in discrete-time LQR (Linear-Quadratic Regulator) 
control of switched linear system. An efficient algorithm, called the Time-Optimal Switching (TOS) algorithm, is proposed for the time-optimal switching control of nonlinear systems with a single control output is considered by Kaya and Noakes in [8]. In [8], firstly, a switching control is found using the STC (Switching Time Computation) method to get from an initial point to a target point with a given number of switchings. Then by means of constrained optimization techniques, the cost being considered as a summation of the arc times, a minimum-time switching control solution is obtained.

The rest of this paper is organized as follows: The problem formulation and certain definitions are given in Section 2, the transformation for the given problem and related theorems are described in Section 3, "Gradient Projection Method Algorithm" for this problem is given in Section 4, numerical results on the example are given in Section 5. Finally, in Section 6 the conclusion of the paper is presented.

\section{PROBlem FORMULATION}

In Kurina and Zhou [10], the authors studied the following minimizing optimal control problem:

Problem I: Minimizing the functional

$$
\begin{aligned}
J\left(u, t_{1}\right)= & \frac{1}{2}\left\langle C_{1} x_{1}\left(t_{1}\right)-C_{2} x_{2}\left(t_{1}\right), F\left(C_{1} x_{1}\left(t_{1}\right)-C_{2} x_{2}\left(t_{1}\right)\right)\right\rangle \\
& +\sum_{j=1}^{2} \int_{t_{j-1}}^{t_{j}}\left(\left\langle x_{j}(t), W_{j}(t) x_{j}(t)\right\rangle+\left\langle u_{j}(t), R_{j}(t) u_{j}(t)\right\rangle\right) d t
\end{aligned}
$$

where, $u=\left(u_{1}, u_{2}\right)$, with respect to the trajectories of the system

$$
\dot{x}_{j}(t)=A_{j}(t) x_{j}(t)+B_{j}(t) u_{j}(t), \quad t_{j-1} \leq t \leq t_{j}, j=1,2
$$

with the following boundaries: $x_{1}(0)=x^{0}, x_{2}(T)=x^{T}$.

Here, $0=t_{0}<t_{1}<t_{2}=T$, the values $t_{0}, t_{2}$ are fixed, $t_{1}$ is not fixed, $x_{j}(t) \in$ $X_{j}, u_{j}(t) \in U_{j}, A_{j}(t), W_{j} \in L\left(X_{j}\right), B_{j}(t) \in L\left(U_{j}, X_{j}\right), R_{j}(t) \in L\left(U_{j}\right)$ for all $t \in$ $\left[t_{j-1}, t_{j}\right], j=1,2 ; C_{1} \in L\left(X_{1}, Y\right), C_{2} \in L\left(X_{2}, Y\right), F \in L(Y), X_{j}, U_{j}, Y$ are real finite dimensional Euclidean spaces, the operators $F, W_{j}(t) \geq 0, R_{j}(t)>0$ for all $t \in\left[t_{j-1}, t_{j}\right] ; x^{0} \in X_{1}, x^{T} \in X_{2}$ are given and symmetric, the operators $F, C_{1}, C_{2}$ are independent of $t$, but the other operators depend continually on $t$ in the corresponding segment $\left[t_{j-1}, t_{j}\right], j=1,2,<., .>$ means an inner product in the appropriate spaces.

Remark 1. In [10], it is assumed that the intermediate point $t_{1}$ is fixed. For this, the minimization functional has the form:

$$
J(u)=\frac{1}{2}\left\langle C_{1} x_{1}\left(t_{1}\right)-C_{2} x_{2}\left(t_{1}\right), F\left(C_{1} x_{1}\left(t_{1}\right)-C_{2} x_{2}\left(t_{1}\right)\right)\right\rangle
$$




$$
+\sum_{j=1}^{2} \int_{t_{j-1}}^{t_{j}}\left(\left\langle x_{j}(t), W_{j}(t) x_{j}(t)\right\rangle+\left\langle u_{j}(t), R_{j}(t) u_{j}(t)\right\rangle\right) d t
$$

i,e., in $[9,10]$, the minimization functional is not depend from the switching point $t_{1}$, because $t_{1}$ is fixed. For this reason in the papers the minimizing functional is written in the form $J(u)$. But in the presented paper, we consider a more general case. It is considered that the point $t_{1}$ is unknown, the minimizing functional has the form as in (2.1), i.e., $J\left(u, t_{1}\right)$. Let us make the following substitution $u(t)=\left(u_{1}(t), u_{2}(t)\right)$ and $x(t)=\left(x_{1}(t), x_{2}(t)\right)$.

Definition 1. The triple $w=\left(t_{1}, u(t), x(t)\right)$ is called admissible, if it satisfies all constraints of Problem I (about the constraints see [10]).

Definition 2. The triple $w^{0}=\left(t_{1}, u(t), x(t)\right)$ is called optimal control, if $J\left(w^{0}\right) \leq$ $J(w)$ for all admissible process $w$.

\section{TRANSFORMATION}

Let us take following transformation. Assume a new parameter $x_{n+1}$ such us satisfies following differential equation with initial condition in the interval $\left[t_{0}, t_{2}\right]$ and $\frac{d x_{n+1}(t)}{d t}=0$ with initial condition $x_{n+1}(0)=t_{1}$. It means that $x_{n+1}$ is constant in $\left[t_{0}, t_{2}\right]$. Next, a new independent time variable $\tau$ is introduced as:

$$
t= \begin{cases}t_{0}+\left(x_{n+1}-t_{0}\right) \tau, & 0 \leq \tau<1 \\ x_{n+1}+\left(t_{2}-x_{n+1}\right)(\tau-1), & 1 \leq \tau \leq 2\end{cases}
$$

then we can write

$$
d t= \begin{cases}\left(x_{n+1}-t_{0}\right) d \tau, & 0 \leq \tau<1 \\ \left(t_{2}-x_{n+1}\right) d \tau, & 1 \leq \tau \leq 2 .\end{cases}
$$

Clearly, (3.1) is a linear mapping with $t: \tau \rightarrow\left[t_{0}, t_{1}\right]$ when $\tau \in[0,1)$ and $t: \tau \rightarrow\left[t_{1}, t_{2}\right]$ when $\tau \in[1,2]$. In fact, $\tau=0$ corresponds to $t=t_{0}, \tau=1$ corresponds to $t=t_{1}$, and $\tau=2$ to $t=t_{2}$. By using relation (3.1) it is easy to introduce the inverse mapping $\tau=\frac{t-t_{0}}{x_{n+1}-t_{0}}$, for $0 \leq \tau \leq 1$ and $\tau=\frac{t-x_{n+1}}{t_{2}-x_{n+1}}$, for $1 \leq \tau \leq 2$. By introducing $x_{n+1}$, $\tau$ and certain substitutions $y_{i}(\tau)=x_{i}(t(\tau)), \quad v_{i}(\tau)=u_{i}(t(\tau)), \quad i=1,2$ and using relation (3.2) the main problem is transcribed into the following equivalent form.

Problem II:

$$
\operatorname{subsystem}(1): \begin{cases}\frac{d y_{1}(\tau)}{d \tau} & =\left(x_{n+1}-t_{0}\right)\left(A_{1}(\tau) y_{1}(\tau)+B_{1}(\tau) v_{1}(\tau)\right) \\ \frac{d x_{n+1}}{d \tau} & =0 \\ x_{n+1}(0) & =t_{1}\end{cases}
$$


in the interval $\tau \in[0,1)$ and

$$
\operatorname{subsystem}(2): \begin{cases}\frac{d y_{2}(\tau)}{d \tau} & =\left(t_{2}-x_{n+1}\right)\left(A_{2}(\tau) y_{2}(\tau)+B_{2}(\tau) v_{2}(\tau)\right) \\ \frac{d x_{n+1}}{d \tau} & =0 \\ x_{n+1}(0) & =t_{1}\end{cases}
$$

in the interval $\tau \in[1,2]$ and the minimizing functional takes the form

$$
\begin{aligned}
\tilde{J}\left(v, x_{n+1}\right)= & \left.\frac{1}{2}\left\langle C_{1} y_{1}(1)-C_{2} y_{2}(1), F\left(C_{1} y_{1}(1)\right)-C_{2} y_{2}(1)\right)\right\rangle \\
& +\int_{0}^{1}\left(x_{n+1}-t_{0}\right)\left(\left\langle y_{1}(\tau), W_{1}(\tau) y_{1}(\tau)\right\rangle+\left\langle v_{1}(\tau), R_{1}(\tau) v_{1}(\tau)\right\rangle\right) d \tau \\
& +\int_{1}^{2}\left(t_{2}-x_{n+1}\right)\left(\left\langle y_{2}(\tau), W_{2}(t) y_{2}(\tau)\right\rangle+\left\langle v_{2}(\tau), R_{2}(\tau) v_{2}(\tau)\right\rangle\right) d \tau .
\end{aligned}
$$

After this transformation we reduce Problem I to Problem II. In Problem II, the state trajectory is $y(\tau)=\left(y_{1}(\tau), y_{2}(\tau)\right)$ and the control tuple is $v(\tau)=$ $\left(v_{1}(\tau), v_{2}(\tau), x_{n+1}\right), \quad 0 \leq \tau \leq 2$.

Since $x_{n+1}$ is an unknown constant (parameter) in the interval [0,2] (see (3.3) and (3.4)), after the transformation, the dimension of Problem II will be the same as the dimension of Problem I.

Theorem 1. There is a one-to-one corresponding between the admissible process $\left(t_{1}, x(t), u(t)\right)$ for Problem I and the admissible process $(y(\tau), v(\tau))$ for Problem II.

Proof. By using transformation from the admissible process $\left(t_{1}, x(t), u(t)\right)$, we obtained admissible process $(y(\tau), v(\tau))$. Let us prove inverse opinion; if $(y(\tau), v(\tau)$ is an admissible process (where $v(\tau)=\left(v_{1}(\tau), v_{2}(\tau)\right.$ ) in problem (3.3)-(3.4), then by using relation (3.1) we can say, if we take $\tau=0$ then $t=t_{0}, \tau=1$ then $t=x_{n+1}$ (in fact $x_{n+1}(0)=t_{1}$ ), and for $\tau=2$ then $t=t_{2}$. It means we obtained intervals $\left[t_{0}, t_{1}\right]$ and $\left[t_{1}, t_{2}\right]$. From relation (3.1), we have $\tau=\frac{t-t_{0}}{x_{n+1}-t_{0}}, 0 \leq \tau \leq 1$ and $\tau=$ $\frac{t-x_{n+1}}{t_{2}-x_{n}+1}, 1 \leq \tau \leq 2$. Then, introducing the notions $x_{1}(t)=y_{1}(\tau(t))$ and $x_{2}(t)=$ $y_{2}(\tau(t))$ we obtain $\dot{x}_{1}=\dot{y}_{1}(\tau(t))\left(\frac{1}{x_{n+1}-t_{0}}\right)$ and $\dot{x}_{2}=\dot{y}_{2}(\tau(t))\left(\frac{1}{t_{2}-x_{n+1}}\right)$ by using the chain rule. If we consider this in (3.3) and (3.4), we can come to the point that $\left(t_{1}, x(t), u(t)\right)$ is the admissible process for the equations (2.1) and (2.2).

Theorem 2. This corresponding mapping between the admissible processes $\left(t_{1}, x(t), u(t)\right)$ and $(y(t), v(t))$ for the equations (2.2), (3.3) and (3.4) preserves the value of the cost functionals (2.1) and (3.5).

Proof. In fact, assume that process $\left(t_{1}^{0}, x^{0}(t), u^{0}(t)\right)$ is an optimal control for Problem I. Let us take process $\left(y^{0}(\tau), v^{0}(\tau)\right)$, which is obtained from the optimal 
process $\left(t_{1}^{0}, x^{0}(t), u^{0}(t)\right)$ of the above mentioned transformation. Assume that $\left(y^{0}(\tau), v^{0}(\tau)\right)$, is not an optimal process and there exists another optimal process $(\tilde{y}(\tau), \tilde{v}(\tau))$ with $\tilde{J}(\tilde{y}(\tau), \tilde{v}(\tau)) \leq J\left(y^{0}(\tau), v^{0}(\tau)\right)$. Take the corresponding admissible process, which is obtained by the inverse transformation from the process $\left(\tilde{x}_{n+1}, \tilde{y}(\tau), \tilde{v}(\tau)\right)$ and denote it by $\left(t_{1}, u(t), x(t)\right)$. Then, it is clear that the cost $J\left(t_{1}, u(t), x(t)\right)=\tilde{J}(\tilde{y}(\tau), \tilde{v}(\tau)) \leq \tilde{J}\left(y^{0}(\tau), v^{0}(\tau)=\tilde{J}\left(t_{1}^{0}, x^{0}(t), u^{0}(t)\right)\right.$. But it contradicts to the optimality of the process $\left(t_{1}^{0}, x^{0}(t), u^{0}(t)\right)$ in Definition 2 . The inverse opinion can be proved in the same way.

Using the theorems, it is straightforward to affirm the following Corollary.

Corollary 1. If the process $\left(t_{1}^{0}, x^{0}(t), u^{0}(t)\right)$ gives minimum for Problem I, then the process $\left(y^{0}(\tau), v^{0}(\tau)\right)$, which is obtained after transformation, gives minimum value for Problem II, and vice versa.

\section{GRADIENT PROJECTION METHOD ALGORITHM}

We have three optimized arguments: First one is the scalar argument $t_{1} \in\left[t_{0}, t_{f}\right]$, the second one is a first control function $v_{1}(t)$ for $t \in\left[t_{0}, t_{m i d}\right]$ and the last one is a second control function $v_{2}(t)$, for $t \in\left[t_{m i d}, t_{f}\right]$. That is $x=\left(t_{1}, v_{1}(t), v_{2}(t)\right)$ with the cost function $J\left(t_{1}, v_{1}(t), v_{2}(t)\right)$ and with the only constraint put on $t_{1}: t_{0} \leq t_{1} \leq t_{f}$.

In the present form, the above admissible process arguments represent an infinitedimensional optimization problem. By applying the "parametrization technique", we can reduce the initial infinite-dimensional optimization problem to a finitedimensional optimization problem. The usefulness of this procedure is that for solution to a finite-dimensional optimization problem there exists a sufficiently powerful arsenal of methods and algorithms.

To convert the problem into a finite-dimensional optimization problem we apply the following parametrization technique: Let's partition the sections $\left[t_{0}, t_{m i d}\right]$ and $\left[t_{\text {mid }}, t_{f}\right]$ into finite number of sub-segments:

$$
\left[t_{0}, t_{\text {mid }}\right]=\bigcup_{i=1}^{N}\left[a_{i}, b_{i}\right) \operatorname{and}\left[t_{\text {mid }}, t_{f}\right]=\bigcup_{j=1}^{M}\left[c_{j}, d_{j}\right) .
$$

Instead of the functions $v_{1}(t)$ and $v_{2}(t)$ we consider their piecewise constant approximations:

$$
\begin{aligned}
& v_{1}(t)=u_{1}^{i}=\text { constant, if } t \in\left[a_{i}, b_{i}\right), i=1,2, \ldots, N ; \\
& v_{2}(t)=u_{2}^{j}=\text { constant, if } t \in\left[c_{j}, d_{j}\right), j=1,2, \ldots, M ;
\end{aligned}
$$

Thus, instead of the admissible process arguments we obtain a finite-dimensional optimization problem:

$$
t_{1}, u_{1}^{i}, u_{2}^{i} \text { with the cost function: } J\left(t_{1} ; u_{1}^{1}, u_{1}^{2}, \ldots, u_{1}^{N} ; u_{2}^{1}, u_{2}^{2}, \ldots, u_{2}^{M}\right) \text {. }
$$


To solve the above finite-dimensional optimization problem we propose to use first-order optimization techniques, i.e. gradient-based methods, e.g. gradient projection procedure. Here are the steps of this procedure:

1) As an initial guess we choose some values for the optimized arguments of the cost function:

$x^{0}=\left(t_{1}^{0}, u_{1}^{1^{0}}, u_{1}^{2^{0}}, \ldots, u_{1}^{N^{0}} ; u_{2}^{1^{0}}, u_{2}^{2^{0}}, \ldots, u_{2}^{M^{0}}\right)$ so that the constraint is satisfied.

2) Then the considered procedure is an ordinary gradient method

$$
x^{k+1}=x^{k}-\alpha_{k} \cdot \nabla f\left(x_{k}\right),
$$

where $\nabla f\left(x_{k}\right)$ is the gradient of the cost functional at the point $x_{k} ; \alpha_{k}$ is the step in the direction of the anti-gradient.

3 ) If after completing the next iteration of (4.1) we trespass the allowable boundaries for the argument $x_{1}^{k+1}$, which in our case is $t_{1}^{k+1}$, we put it back into $\left[t_{0}, t_{f}\right]$ according to the following formula:

$$
t_{1}^{k+1}= \begin{cases}0, & t_{1}^{k+1}<0 \\ 2, & t_{1}^{k+1}>2\end{cases}
$$

4) We repeat steps $2-3$ for new $k:=k+1$ until some exit criterion is satisfied. Possible exit criterions:
- $\left\|\nabla f\left(x_{k}\right)\right\| \leq \epsilon_{1}$

$$
\text { - }\left|x^{k+1}-x^{k}\right|<\epsilon_{3}
$$$$
\left|f\left(x^{k+1}\right)-f\left(x^{k}\right)\right|<\epsilon_{2}
$$

\section{EXAMPLE}

In this paper, inspired by [9], we consider the switching point $t_{1}$ as non fixed. Then, we will try to reduce the unknown switching case to the known switching case, after which all the procedure in [10] can be used. Consider the following problem of minimizing the functional,

$$
\begin{aligned}
J\left(x, u_{1}, u_{2}, t_{1}\right)= & \frac{1}{2}\left[\left(x_{11}\left(t_{1}\right)+x_{21}\left(t_{1}\right)\right)^{2}+\int_{0}^{t_{1}}\left(x_{11}^{2}(t)+2 x_{11}(t) x_{12}(t)\right.\right. \\
& \left.+3 x_{12}^{2}(t)+u_{1}^{2}(t)\right) d t+\int_{t_{1}}^{2}\left(x_{21}^{2}(t)+8 x_{22}^{2}(t)\right. \\
& \left.\left.+u_{2}^{2}(t)\right) d t\right]
\end{aligned}
$$

with respect the trajectories of the systems

$$
\begin{aligned}
& \operatorname{subsystem}(1):\left\{\begin{array}{l}
\dot{x}_{11}(t)-x_{11}(t)=0 \\
x_{12}(t)+u_{1}(t)=0 \\
x_{11}(0)
\end{array}=-1, \text { for } t \in\left[0, t_{1}\right)\right.
\end{aligned}
$$

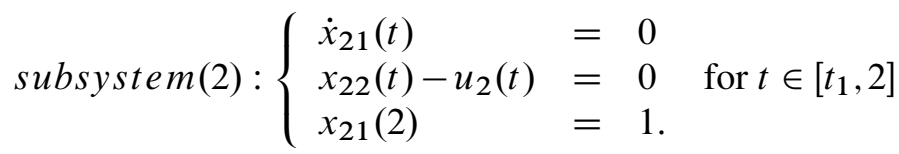


We will use transformation (3.1) which is reduced problem (5.2), (5.3) to the new problem without unknown switching point. For this aim, take new variable $\dot{x}_{n+1}(t)=0, x_{n+1}(0)=t_{1}$. From this differential equation, it is clear $x_{n+1}=t_{1}$ is unknown constant in [0,2]. Take also the state trajectories $y_{i, j}(\tau)=x_{i, j}(t(\tau))$, and controls $v_{i}(\tau)=u_{i}(t(\tau))$ where $i, j=1,2$. Let us also use interval transformation in (3.1) with $t_{0}=0$ and $t_{2}=2$. Then we can come the point that, if $\tau=0$ then $t=0$, if $\tau=1$ then $t=x_{n+1}=t_{1}$, and, if $\tau=2$ then $t=2$. If we use all these transformations, then the minimizing functional and the state equations will take the following form:

$$
\begin{aligned}
J(v)= & \frac{1}{2}\left[\left(y_{11}(1)+y_{21}(1)\right)^{2}+t_{1} \int_{0}^{1}\left(y_{11}^{2}(\tau)+2 y_{11}(\tau) y_{21}(\tau)+3 y_{12}^{2}\right.\right. \\
& \left.\left.+v_{1}^{2}(\tau)\right) d \tau+\left(2-t_{1}\right) \int_{1}^{2}\left(y_{21}^{2}(\tau)+8 y_{22}^{2}(\tau)+v_{2}^{2}(\tau)\right) d \tau\right]
\end{aligned}
$$

where, $v=\left(v_{1}, v_{2}\right)$, and state equations takes the form

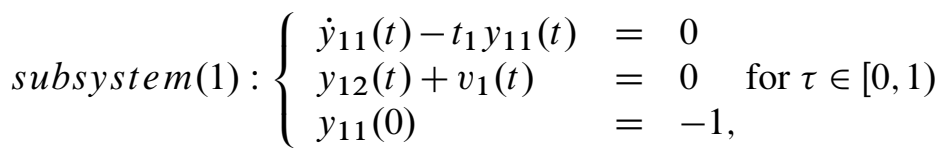

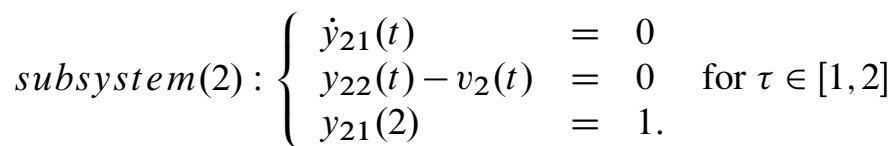

If we solve (5.5) with respect to the states $y_{11}(t)$ and $y_{12}(t)$ and handle (5.6) with respect to the states $y_{21}(t)$ and $y_{22}(t)$, then putting these in (5.4), the functional cost gets the form:

$$
\begin{aligned}
J\left(t_{1}, v_{1}, v_{2}\right)= & \frac{1}{2}\left[\left(1-\exp \left(t_{1}\right)\right)^{2}+t_{1} \int_{0}^{1}\left(\exp \left(2 t_{1} \tau\right)+2 \exp \left(t_{1} \tau\right) v_{1}(\tau)\right.\right. \\
& \left.\left.+4 v_{1}^{2}(\tau)\right) d \tau+\left(2-t_{1}\right) \int_{1}^{2}\left(1+9 v_{2}^{2}(\tau)\right) d \tau\right]
\end{aligned}
$$

To solve (5.7) by finite-optimization techniques first we transform the functional into finite-dimensional problem as follows:

$$
\begin{aligned}
J\left(t_{1}, w_{1}, w_{2}\right)= & \frac{1}{2}\left[\left(1-\exp \left(t_{1}\right)\right)^{2}+t_{1} \sum_{i=1}^{N} \int_{0}^{1}\left(\exp \left(2 t_{1} \tau\right)+2 \exp \left(t_{1} \tau\right) w_{1}^{i}(\tau)\right.\right. \\
& \left.\left.+4\left(w_{1}^{i}\right)^{2}(\tau)\right) d \tau+\left(2-t_{1}\right) \sum_{j=1}^{M} \int_{1}^{2}\left(1+9\left(w_{2}^{j}\right)^{2}(\tau)\right) d \tau\right]
\end{aligned}
$$

where, $v_{1}(t)=w_{1}^{i}=$ constant, if $t \in[0,1) ; v_{2}(t)=w_{2}^{j}=$ constant, if $t \in[1,2]$. Then, by using "Gradient Projection Method" we can obtain the following optimal 


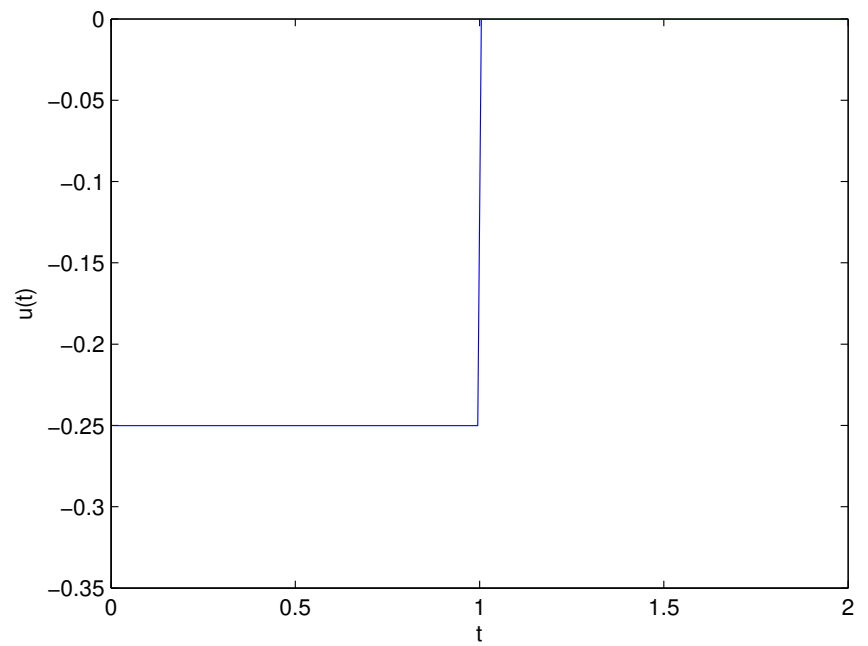

FIGURE 1. Optimal Control Input

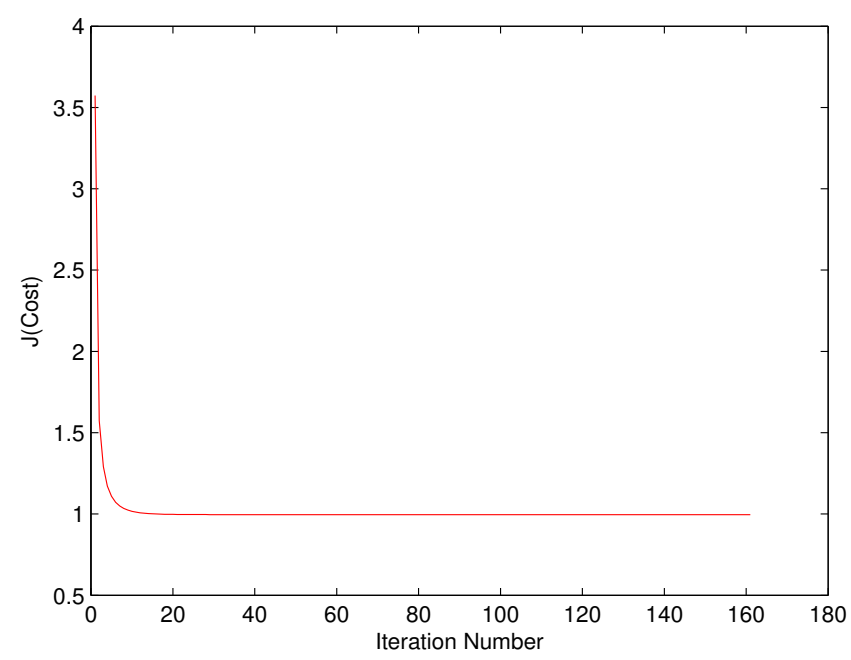

FIGURE 2. Optimal Cost

control input and state variable histories numerically (Figures 1 and 2). By applying Gradient Algorithm for the initial nominal $t_{1}=1.0$, after 160 iterations we find that the optimal switching time $t_{1}^{*}=0.0653$ and the optimal cost $J^{*}=0.9958$. The 
computation takes about 0.7387 seconds of CPU time using C Sharp as programming language on an Intel(R)Core(TM)i7-3720QM $2.60 \mathrm{GHz}$ PC with 8GB of RAM.

\section{CONCLUSION}

In this paper we obtained the approach for optimal switching control problem with unknown switching points which described in $[9,10]$. At that case, switching point $t_{1}$ admitted as unknown and unfixed point in the known interval for state equations and unknown boundary of the integral. Moreover, the cost functional components was transcribed by the linear transformation and the system was solved by using Gradient Projection Method numerically.

It is also possible to say for the future works that if there are $K$ numbers of switchings, then it is no difficulty in applying the previous method to the problems with several subsystems. If there exist non fixed switchings, $t_{0}, t_{1}, t_{2}, \ldots, t_{K}$ and $T=0$ with $0=t_{0}<t_{1}<t_{2}<, \ldots,<t_{K}<T=0$, then we can transcribe the problem into an equivalent problem by introducing $K$ new state variables $x_{n+1}, x_{n+2}, \ldots, x_{n+K}$ which correspond to the switching instants $t_{1}, t_{2}, \ldots, t_{K}$ and satisfy the following equations: $\frac{d x_{n+i}}{d \tau}=0, \quad x_{n+i}(0)=t_{i}, \quad \tau \in[1,2], \quad i=1,2, \ldots, K$.

The new independent time variable $\tau$ has a linear relationships with $t$ where $\tau=0$ corresponds to $t=t_{0}, \quad \tau=1$ corresponds to $t=t_{1}, \ldots, \tau=K+1$ corresponds to $t=t_{T}$.

\section{ACKNOWLEDGEMENT}

The authors would like to thank the editor, the associate editors, and anonymous referees for their constructive corrections and valuable suggestions that improved the manuscript considerably.

\section{REFERENCES}

[1] L. Y. Wang, A. Beydoun, J. Cook, J. Sun, and I. Kolmanovsky, "Optimal hybrid control with applications to automotive powertrain systems." Control and Information Sciences, vol. 222, no. 1, pp. 190-200, 1997, doi: 10.1007/BFb0036095.

[2] V. V. Azmyakov, R. G. Guerra, and A. E. Polyakov, " On the method of dynamic programming for linear-quadratic problems of optimal control in hybrid systems." Automation and Remote Control, vol. 70, no. 5, pp. 787-799, 2009, doi: 10.1134/S0005117909050075.

[3] A. Bensoussan and J. Menaldi, "Dynamics of Continuous, Discrete and Impulsive Systems Series B: Application and Algorithm." Hybrid Control and dynamic programming, vol. 3, no. 4, pp. 395-442, 1997, doi: 10.1109/JPROC.2000.871299.

[4] V. Boltyanskii, "The maximum principle for variable structure systems." International Journal of Control, vol. 7, no. 7, pp. 1445-1451, 2004, doi: 10.1080/00207170412331319312.

[5] M. S. Branicky, V. S. Borkar, and S. K. Mitter, " A unifed framework for hybrid control: model and optimal control theory." IEEE Transactions on Automatic Control , vol. 43, no. 1, pp. 31-45, 1998, doi: 10.1109/9.654885.

[6] I. Capuzzo Dolcetta, "Optimal switching for ordinary diferential equations." SIAM Journal of Control and Optimization, vol. 22, no. 1, pp. 143-161, 1984, doi: 10.1137/0322011. 
[7] A. V. Dmitruk and A. M. Kaganovich, "The Hybrid Maximum Principle is a Consequence of Pontryagin Maximum Principle.” System and Control Letters, vol. 57, no. 11, pp. 964-970, 2008, doi: 10.1016/j.sysconle.2008.05.006.

[8] C. Y. Kaya and L. J. Noakes, "Computational Method for Time-Optimal Switching Control." Journal of Optimization Theory and Applications, vol. 117, no. 1, pp. 69-92, 2003, doi: 1023/A:1023600422807.

[9] G. A. Kurina, "On Decomposition of Linear-Quadratic Optimal Control Problems for Two-Steps Descriptor Systems." 50th IEEE Conference on Decision and Control and European Control Conference, pp. 6705-6711, 2011, doi: 10.1109/CDC.2011.6160984.

[10] G. A. Kurina and Y. Zhou, "Optimal Control problems for Two-Steps Systems." Doklady Mathematics, vol. 83, no. 2, pp. 275-277, 2011, doi: 10.1134/S1064562411020049.

[11] R. Li, K. L. Teo, K. H. Wong, and G. R. Duan, " Control parameterization enhancing transform for optimal control of switched systems." Mathematical and Computer Modelling, vol. 43, no. 11, pp. 1993-1403, 2006, doi: 10.1016/j.mcm.2005.08.012.

[12] B. Lincoln and B. M. Bernhardsson, "Efficient pruning of search trees in lqr control of switched linear systems." In Proceedings of the 39th IEEE Conference on Decision and Control , pp. 18281833, 2000, doi: 10.1109/CDC.2000.912128.

[13] J. Lu, L. Z. Liao, A. Nerode, and J. H. Taylor, "Optimal control of systems with continuous and discrete states." In Proceedings of the 32nd IEEE Conference on Decision and Control, pp. 22922297, 1993, doi: 10.1109/CDC.1993.325606.

[14] S. K. Lucas and C. Y. Kaya, "Switching-time computation for bang-bang control laws." American Control Conference, 2001. Proceedings of the 2001 , pp. 176-181, 2001, doi: 10.1109/ACC.2001.945537.

[15] G. Ma, Y. Zhang, and M. Liu, “ A generalized gradient projection method based on a new working set for minimax optimization problems with inequality constraints.” J. Inequal Appl., pp. 18281833, 2017, doi: 10.1186/s13660-017-1321-3.

[16] S. F. Maharramov, "Optimality condition of a nonsmooth switching control system." Automatic Control and Computer Sciences, vol. 42, no. 2, pp. 94-101, 2008, doi: 10.3103/S0146411608020077.

[17] S. F. Maharramov and K. B. Mansimov, "Optimization of a class of discrete step control systems." Comput. Math. Math. Phys., vol. 41, no. 3, pp. 334-339, 2001, doi: 10.3103/S0146411608020077.

[18] B. Piccoli, "Hybrid systems and optimal control." In Proceedings of the 37th IEEE Conference on Decision and Control, vol. 4, pp. 13-18, 1998, doi: 10.1109/CDC.1998.760582.

[19] H. J. Sussmann, “A maximum principle for hybrid optimal control problems." In Proceedings of the 38th IEEE Conference on Decision and Control, 1999, doi: 10.1109/CDC.1999.832814.

[20] H. S. Witsenhausen, "A class of hybrid-state continuous-time dynamic systems." IEEE Transactions on Automatic Control, vol. 11, no. 2, pp. 161-167, 1966, doi: 10.1109/TAC.1966.1098336.

[21] H. Xu, L. Xinzhi, and K. L. Teo, " Delay independent stability criteria of impulsive switched systems with time-invariant delays." Mathematical and Computer Modelling, vol. 47, no. 3, pp. 372-379, 2008, doi: 10.1016/j.mcm.2007.04.011.

[22] J. Yong, "Systems governed by ordinary diferential equations with continuous." Applied Mathematics and Optimization, vol. 20, no. 1, pp. 223-235, 1989, doi: 10.1007/BF01447655.

Authors' addresses

Shahlar Meherrem

Department of Mathematics, Yasar University, Izmir, Turkey,

E-mail address: sahlar.meherremeyasar.edu.tr 
Deniz H. Gucoglu

Department of Mathematics, Yasar University, Izmir, Turkey,

E-mail address: denizhasan09@gmail.com

Samir Guliyev

Azerbaijan State Oil \& Industry University, Institue of Control Systems of ANAS, Baku, Azerbaijan,

E-mail address: azcopal@gmail.com 